\title{
Anharmonicity, vibrational instability, and the Boson peak in glasses
}

\author{
V. L. Gurevich \\ Solid State Physics Division, A. F. Ioffe Institute, 194021, Saint Petersburg, Russia \\ D. A. Parshin \\ Saint Petersburg State Technical University, 195251, Saint Petersburg, Russia
}

H. R. Schober

Institut für Festkörperforschung, Forschungszentrum Jülich, D-52425, Jülich, Germany

(Received 30 July 2002; revised manuscript received 2 January 2003; published 17 March 2003)

\begin{abstract}
We show that a vibrational instability of the spectrum of weakly interacting quasilocal harmonic modes creates the maximum in the inelastic scattering intensity in glasses, the Boson peak. The instability, limited by anharmonicity, causes a complete reconstruction of the vibrational density of states (DOS) below some frequency $\omega_{c}$, proportional to the strength of interaction. The DOS of the new harmonic modes is independent of the actual value of the anharmonicity. It is a universal function of frequency depending on a single parameter - the Boson peak frequency $\omega_{b}$ which is a function of interaction strength. The excess of the DOS over the Debye value is $\propto \omega^{4}$ at low frequencies and linear in $\omega$ in the interval $\omega_{b} \ll \omega \ll \omega_{c}$. Our results are in an excellent agreement with recent experimental studies.
\end{abstract}

DOI: 10.1103/PhysRevB.67.094203

PACS number(s): 61.43.Fs, 63.50.+x, 78.30.Ly

\section{INTRODUCTION}

One of the most striking properties of glasses is a maximum in the inelastic scattering intensity observed in neutron and Raman scattering at frequencies between 0.5 and $2 \mathrm{THz}$, far below the Debye frequency. ${ }^{1}$ This so called Boson peak (BP) indicates an excess of low frequency vibrations over the Debye value which is given by the sound waves. It is seen as a maximum in the vibrational density of states (DOS) divided by $\omega^{2}, G(\omega) / \omega^{2}$ but not necessarily in $G(\omega)$ itself. $^{2}$ The BP is a general feature, its magnitude varies strongly between materials.

Despite numerous efforts, the BP remains one of the most intriguing problems of solid state physics. Some authors attribute the BP to vibrations of clusters of atoms of typical sizes. ${ }^{3,4}$ The physical origin of these clusters in homogeneous amorphous media remains unclear and they have not been identified in numerical simulations.

Another popular qualitative explanation of the Boson peak is a softening of acoustic phonons by static disorder ${ }^{5,6}$ due to elastic Rayleigh scattering. However, even the most optimistic estimates show that the Rayleigh contribution is at least 4 times too small to explain the experimental data on thermal conductivity in glasses. ${ }^{5}$ This mechanism is also in contradiction to the linear dispersion law for acoustical phonons at the Boson peak frequency seen in molecular dynamics in Ref. 7.

Sometimes the BP is related to low lying optic modes of parental crystals. ${ }^{8-10}$ Whereas the BP is a general feature of glasses such crystal structures with soft optic modes cannot be identified always. Such a mechanism is possible in some cases. Yet it remains a puzzle how these crystalline peaks are transformed in the glassy state to a shoulder in the vibrational DOS.

Recent work on harmonic lattice models demonstrated that softening of disordered force constants can smear and push to low frequencies peaks which exist in the crystalline DOS. ${ }^{11-13}$ In another approach the vibrations of a random distribution of atoms, interacting with a Gaussian-shaped pair potential, was studied ${ }^{14}$ in a harmonic scalar approximation. Reducing the density the system becomes unstable. Approaching this instability a low frequency peak appears in $G(\omega) / \omega^{2}$ which resembles the BP. The main drawback of these models is their neglect of the static displacement of the atoms in response to disorder. In real glasses where short range order is conserved such feedback always occurs since the forces between the atoms strongly depend on their distance. In particular, the above models have no built in mechanism to stabilize vibrations with negative $\omega^{2}$, unstable modes.

The proposed models of the Boson peak do not account for anharmonicity effects which, as we will show in the present paper, become very important especially for small force constants. Glasses at low frequencies and temperatures are highly anharmonic as seen in most of their macroscopic thermodynamic functions. Anharmonicity and static displacements, together, stabilize otherwise unstable vibrational modes. This does not however, imply that the vibrations at the Boson peak are anharmonic. Anharmonicity is essential in forming the equilibrium structure, and thus the force constants which determine the proper harmonic spectrum of low frequency modes.

Another important point is that the previous explanations do not relate the Boson peak, which is one of the universal properties of glasses, to other universal properties, such as the two-level systems which dominate the low-temperature behavior or the plateau in the thermal conductivity at moderate temperatures. This relationship naturally emerges in our approach.

\section{QUASILOCALIZED VIBRATIONS (QLV'S)}

We present a universal mechanism for the formation of a $\mathrm{BP}$ in glasses, out of an originally flat DOS. This arises from 
the following three features: (1) quasilocal vibrations (QLV's) (resonant states) with a smooth, structureless initial DOS, $g_{0}(\omega)$ at low $\omega$, (2) an elastic interaction between them, and (3) stabilization by anharmonicity when the system becomes unstable because of interaction.

Similar to the well known two level systems, the QLV's are a typical feature of disordered systems. They are additional modes and are characterized by a large vibrational amplitude of some group of atoms. Their existence in glasses was predicted in Refs. 15-17. They can be described as low frequency harmonic oscillators (HO's) which couple bilinearly to the sound waves, see Refs. 18-20. This in turn leads to a dipole-dipole interaction between different HO's. The importance of the elastic interaction between local defects in glasses has been stressed by $\mathrm{Yu}$ and Leggett ${ }^{21}$ and Grannan et $a l^{22}$

The microscopic origin of QLV's in disordered systems varies and depends on the type of disorder. Broadly speaking, the QLV's can be divided into two groups. First there are materials where QLV's exist independently of the structural disorder typical for structural glasses and amorphous solids. Orientationally disordered ("plastic") crystals belong to this group. ${ }^{23-25}$ In these materials some molecular groups librate with low frequencies. In harmonic approximation these soft librations can be identified with QLV's. The local potentials for the librational motion can vary from site to site. In this case we have a distribution of the librational frequencies. The librations couple to the sound waves which in turn induces an interaction between them. ${ }^{22}$ It depends on details of the material whether the interaction is strong enough to reconstruct the original spectrum of QLV's completely or only partially. This effect is seen in recent measurements of dielectrtric loss spectra of orthocarborane. ${ }^{26}$ Similarly coordination defects in covalent materials can lead to QLV as was observed by Biswas et al. ${ }^{27}$ in a simulation of amorphous $\mathrm{Si}$ (see also recent works in Refs. 28 and 29). QLV can originate from numerous defects such as off center ions or interstitial atoms. Depending on the "size" of the defect the QLV's involve more or fewer atoms. Interstitial atoms are the prototype of a topological point defect. QLV's of interstitial atoms in fcc metals were studied extensively in the past, see Ref. 30 for a review. These QLV's have effective masses of four atomic masses and the crystal structure is strongly distorted by the defect and the low frequency of the librational QLV can be traced to the local strain. Low concentrations of these interstitials are already sufficient to destroy the crystalline structure completely. This is utilized in the interstitialcy model of glass formation. ${ }^{31}$

This leads directly to the second group where the QLV's result directly from disorder. Such modes are regularly found in computer simulations, e.g., for soft spheres, ${ }^{32} \mathrm{SiO}_{2},{ }^{33}$ $\mathrm{Se},{ }^{34} \mathrm{Ni}-\mathrm{Zr},{ }^{35} \mathrm{Pd}-\mathrm{Si}$ and $\mathrm{Au}-\mathrm{Si},{ }^{36} \mathrm{NiB},{ }^{37}$ in amorphous ice, ${ }^{38}$ in amorphous and quasicrystalline $\mathrm{Al}-\mathrm{Zn}-\mathrm{Mg},{ }^{39}$ and in simple dense fluids. ${ }^{40}$

In these simulations the QLV's were observed as localized vibrations with frequencies below the minimal sound wave frequency allowed by the size of the simulated sample. A simple indicator of these "size-localized" QLV's is the scaling of the participation ratio with system size. Increasing the system size the minimal sound wave frequency drops and the QLV's are no longer localized in the simulation but show the typical properties of resonant modes, i.e., of low frequency local vibrations which couple bilinearly to the sound waves. The exact eigenvectors of the interacting system of QLV's and sound waves are superpositions of these two types of modes. The effect of system size on the appearance of QLV's in simulations was discussed in detail in Ref. 41. There it was shown that the exact eigenvectors at frequencies up to and above the boson peak can be decomposed into extended sound-wave-like modes and the local cores of QLV's. The latter correspond to the harmonic oscillators of this work.

The physical origin of these disorder induced QLV's can be traced to local irregularities of the amorphous structure. In dense packed metallic glasses these originate, e.g., from the conflict of the local dense packing (icosahedral packing) and global dense packing (fcc or hcp). ${ }^{42}$ These local irregularities can be seen as centers of local strains. ${ }^{43}$

We expect such local strain centers to be ubiquitous. The strains will have broad distributions, which will lead to broad distributions of QLV frequencies. Whereas local strains and QLV's will be a general property of glasses the atomistic structure of QLV's reflects the structure of the considered material. In dense packed metallic systems the cores of the QLV have been found to be chainlike. ${ }^{44,41}$ In $\mathrm{SiO}_{2}$ they are formed by a coupled rotations of $\mathrm{SiO}_{4}$ tetrahedra. ${ }^{17,45} \mathrm{In} \mathrm{Se}$ one has coupled chains and rings, ${ }^{34}$ etc.

Another possible (and natural) mechanism of the QLV formation are low lying optical modes in parental crystals. Disorder in amorphous material would destroy the long range coherence of optical modes. This makes them practically indistinguishable from quasilocal modes.

Together with the tunneling systems, the QLV's form the main ingredient of the soft potential model ${ }^{46,47}$ (see Ref. 48). They manifest themselves in experimental values, e.g., the excess specific heat ${ }^{47,49-51}$ and the plateau in the thermal conductivity, ${ }^{20,52}$ in inelastic light ${ }^{53}$ and neutron scattering, ${ }^{17}$ and they are observed in many numerical calculations, cited above.

\section{DENSITY OF STATES OF QUASILOCALIZED VIBRATIONS}

One might think a priori that the QLV's can have an arbitrary DOS, $g(\omega)$, depending on the particulars of the glass. We will show, that due to the interaction between the HO's, $g(\omega)$ is a universal function at low frequencies. This universality stems from the vibrational instability of the spectrum which occurs in nearly all systems of interacting HO's. Anharmonicity stabilizes the system in new minima, and thus reconstructs the DOS to a new harmonic spectrum. As a result $g(\omega) / \omega^{2}$ acquires a maximum without a peak in $g(\omega)$ itself.

Below we will derive the following form of the reduced DOS of these harmonic resonant modes (excluding the Debye part at low $\omega$ which is not seen in Raman scattering) 


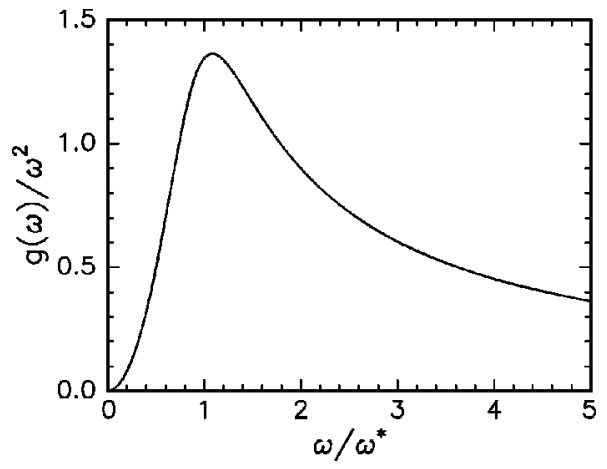

FIG. 1. The Boson peak, Eq. (1).

$$
\begin{aligned}
\frac{g(\omega)}{\omega^{2}}= & \frac{3 C}{\pi \omega^{\star}}\left(\frac{\omega^{\star}}{\omega}\right)^{4}\left[z_{1}^{2}(\omega)+z_{2}^{2}(\omega)\right]^{-1} \\
& \times\left[\frac{1}{2 z_{1}(\omega)} \ln \frac{z_{1}(\omega)+1}{z_{1}(\omega)-1}+\frac{1}{z_{2}(\omega)} \tan ^{-1} \frac{1}{z_{2}(\omega)}\right],
\end{aligned}
$$

where $C$ is a constant $[\mathrm{Eq} .(22)]$ and

$$
z_{1,2}(\omega)=\frac{1}{2} \sqrt{\sqrt{9+8\left(\omega^{\star} / \omega\right)^{6}} \pm 3}
$$

The function $g(\omega) / \omega^{2}$ is plotted in Fig. 1. It depends on a single parameter, $\omega^{\star}$ characterizing the position of the Boson peak. The maximum of $g(\omega) / \omega^{2}$, the Boson peak, is at $\omega_{b}$ $\approx 1.1 \omega^{*}$. For small frequencies, $\omega \ll \omega_{b}, g(\omega) \propto \omega^{4}$ while for large ones, $\omega \gg \omega_{b}, g(\omega) \propto \omega$.

\section{VIBRATIONAL INSTABILITY}

To illustrate our central idea of a vibrational instability, we start with a pair of interacting HO's immersed in an elastic continuum. The potential energy is given by

$$
U_{\text {har }}\left(x_{1}, x_{2}\right)=M_{1} \omega_{1}^{2} x_{1}^{2} / 2+M_{2} \omega_{2}^{2} x_{2}^{2} / 2-I_{12} x_{1} x_{2} .
$$

Here $x_{1,2}$ are the $\mathrm{HO}$ coordinates, $M_{1,2}$ the masses, and $\omega_{1,2}$ the bare frequencies of the two HO's, i.e., neglecting the bilinear interaction. The interaction strength is given by ${ }^{53}$

$$
I_{12}=g_{12} J / r_{12}^{3}, \quad J \equiv \Lambda^{2} / \rho v^{2}
$$

where $g_{12}$ accounts for the relative orientation of the HO's, $r_{12}$ is their distance, $\rho$ is the mass density of the glass, and $v$ is a sound velocity. The interaction between the HO's is due to the coupling between a single $\mathrm{HO}$ and the surrounding elastic medium (the glass). This HO-phonon coupling has the form $^{20}$

$$
\mathcal{H}_{\text {int }}=\Lambda x \varepsilon,
$$

where $\Lambda$ is the coupling constant and $\varepsilon$ the strain.

Diagonalization of Eq. (3) yields two frequencies

$$
\tilde{\omega}_{1,2}^{2}=\frac{\omega_{1}^{2}+\omega_{2}^{2}}{2} \mp \sqrt{\left(\frac{\omega_{1}^{2}-\omega_{2}^{2}}{2}\right)^{2}+\frac{I_{12}^{2}}{M_{1} M_{2}}} .
$$

The smaller value $\tilde{\omega}_{1}^{2}$ becomes negative when the interaction $I_{12} \equiv I$ exceeds the threshold (critical) value

$$
I_{c} \equiv \omega_{1} \omega_{2} \sqrt{M_{1} M_{2}} .
$$

A negative frequency squared indicates an instability of the corresponding eigenstate. At this instability the stable equilibrium point at the origin turns into a saddle point of the potential energy, Eq. (3).

This instability persists also in a system of many interacting HO's. In a real physical system, anharmonic forces always stabilize an embedded $\mathrm{HO}$ in a nearby minimum of the potential energy. The position of this minimum depends on the interaction between HO's. We are thus confronted with the many-body problem of finding the minima of the potential energy for a system of interacting anharmonic oscillators, similar to the one considered in Refs. 22,54. The frequencies in these minima are real and different from the original ones. The harmonic vibrational spectrum is reconstructed. We will call this anharmonicity limited vibrational instability.

\section{STABILIZATION BY ANHARMONICITY}

We will now show that for weak interaction $I$ the reconstructed DOS has, below a characteristic frequency $\omega_{c} \propto|I|$, a universal form irrespective of its original form. First, due to interaction, it becomes a linear function of frequency $g(\omega) \propto \omega$. Secondly, the displacements of the previously unstable oscillators from their old equilibrium positions create static random forces which cause a second reconstruction of the DOS below another frequency $\omega_{b} \ll \omega_{c}$. Due to so called sea-gull singularity ${ }^{47}$ at $\omega=0$ the linear DOS is reconstructed to $g(\omega) \propto \omega^{4}$ for $\omega \ll \omega_{b}$. Together, these two reconstructions produce a maximum of $g(\omega) / \omega^{2}$ at $\omega=\omega_{b}$.

Let us consider a number of randomly distributed, interacting $\mathrm{HO}$ with concentration $n_{0}$ and an initial DOS $g_{0}(\omega)$ (normalized to unity) in the frequency range from 0 to $\omega_{0}$, where $g_{0}(\omega)$ is a monotonously increasing function of $\omega$. For the harmonic part of the interaction we take the generalization of Eq. (3) and add an anharmonic term to stabilize the system

$$
U_{\text {anhar }}=(1 / 4) \sum_{i} A_{i} x_{i}^{4}, \quad A_{i}>0 .
$$

We will take the interaction $I$ to be the small parameter of our theory, i.e., we assume that the typical random interaction I between neighboring HO's is much smaller than the typical values of $M \omega_{0}^{2}$. As $|I| \ll M \omega_{0}^{2}$ frequencies of order $\omega_{0}$ will be practically unaffected by the interaction whereas HO's with frequencies $\omega<\omega_{c}$ will be displaced to new minima, where

$$
\omega_{c} \simeq|I| / M \omega_{0} \ll \omega_{0} .
$$

Since the concentration of unstable HO's is much smaller than the one of the stable ones a low frequency oscillator is typically surrounded by high frequency ones. We can simplify our consideration by again considering pairs of HO's, one with a low frequency $\omega_{1} \lesssim \omega_{c}$ and the other one from the 
bulk of the HO's with a frequency $\omega_{2}$ of the order of $\omega_{0}$ (see Appendix A for the general case). Due to the combined action of interaction and anharmonicity the two HO's will be displaced into new minima $x_{10}$ and $x_{20}$ given by equations $\left(I_{12} \equiv I\right)$

$$
\begin{aligned}
& I x_{20}=x_{10}\left(M_{1} \omega_{1}^{2}+A_{1} x_{10}^{2}\right), \\
& I x_{10}=x_{20}\left(M_{2} \omega_{2}^{2}+A_{2} x_{20}^{2}\right) .
\end{aligned}
$$

For $|I|>I_{c}$ we need the nonzero solutions of these equations. Expanding around either minimum we find the new (harmonic) frequencies from the secular equation

$$
\left|\begin{array}{cc}
\alpha_{1}-M_{1} \omega^{2} & -I \\
-I & \alpha_{2}-M_{2} \omega^{2}
\end{array}\right|=0
$$

with

$$
\alpha_{i}=M_{i} \omega_{i}^{2}+3 A_{i} x_{i 0}^{2}, \quad i=1,2 .
$$

From the condition $\omega_{1} \ll \omega_{2}$ follows $x_{20} \ll x_{10}$ and, therefore, the term $A_{2} x_{20}^{2}$ in Eq. (9) can be neglected giving

$$
x_{20}=\left(I / M_{2} \omega_{2}^{2}\right) x_{10}
$$

and

$$
x_{10}=\omega_{1} \sqrt{M_{1} / A_{1}} \sqrt{\left(I / I_{c}\right)^{2}-1} .
$$

As a result we get from Eq. (10) under the condition $\omega_{1} / \omega_{2} \ll 1$ with this accuracy the new frequencies $\tilde{\omega}_{2}=\omega_{2}$ and

$$
\widetilde{\omega}_{1}^{2}=2 \omega_{1}^{2}\left[\left(I / I_{c}\right)^{2}-1\right] .
$$

The smaller frequency (14) is the solution of the linear equation [compare with Eq. (10)]

$$
M_{2} \omega_{2}^{2}\left(\alpha_{1}-M_{1} \omega^{2}\right)=I^{2} .
$$

It is remarkable that for weak interaction the strength of the anharmonicity $A_{i}$ does not enter the renormalized frequency (14).

Near the threshold where $\left(|I|-I_{c}\right) / I_{c} \ll 1$, the smaller frequency squared $\tilde{\omega}_{1}^{2}$ is proportional to $\left(|I|-I_{c}\right) / I_{c}$. Provided the distribution of the random quantity $I$ is smooth one gets, therefore, below $\omega_{c}$ a linear DOS $[\tilde{g}(\omega) \propto \omega]$ irrespective of the initial form of $g_{0}(\omega)$. In Appendix A it is shown that the same result holds if one has a low frequency $\mathrm{HO}$ surrounded by several high frequency ones. Our numerical calculations (see Sec. VII) also show that this case is typical.

\section{THE BOSON PEAK}

If the low-frequency $\mathrm{HO}$ with their reconstructed linear DOS were isolated, the problem would be solved. There is, however, a further interaction between these oscillators which we have not taken into account so far. The lowfrequency HO's, displaced from their equilibrium positions, create random static forces $f$. The force $f_{i}$ exerted on the $i$ th oscillator by the $j$ th one is

$$
f_{i}=I_{i j} x_{j 0}
$$

In a purely harmonic case, these linear forces would not affect the frequencies. Anharmonicity, however, renormalizes the low frequency part of the spectrum, ${ }^{47}$ a manifestation of the sea-gull singularity. Consider an anharmonic oscillator under the action of a random static force $f$

$$
U(x)=A x^{4} / 4+M \omega_{1}^{2} x^{2} / 2-f x,
$$

where $\omega_{1}$ is the oscillator frequency in the harmonic approximation. The force $f$ shifts the equilibrium position from $x=0$ to $x_{0} \neq 0$, given by

$$
A x_{0}^{3}+M \omega_{1}^{2} x_{0}-f=0,
$$

where the oscillator has a new (harmonic) frequency

$$
\omega_{\text {new }}^{2}=\omega_{1}^{2}+3 A x_{0}^{2} / M .
$$

If $\widetilde{g}_{1}\left(\omega_{1}\right)$ is the distribution function of frequencies $\omega_{1}$ and $P(f)$ is the distribution of random forces, then the renormalized DOS is given by

$$
g(\omega)=\int_{0}^{\infty} \widetilde{g}_{1}\left(\omega_{1}\right) d \omega_{1} \int_{-\infty}^{\infty} d f P(f) \delta\left(\omega-\omega_{\text {new }}\right) .
$$

As the forces between the HO's are proportional to $r_{i j}^{-3}$ their sum is Lorentzian distributed (see Appendix B):

$$
P(f)=\frac{1}{\pi} \frac{\delta f}{f^{2}+(\delta f)^{2}} .
$$

Assuming $\omega \ll \omega_{c}$ and integrating Eq. (20) with $\tilde{g}_{1}\left(\omega_{1}\right)$ $=C \omega_{1}$ we arrive at the integral

$$
\frac{g(\omega)}{\omega^{2}}=\frac{6 C}{\pi \omega^{\star}}\left(\frac{\omega}{\omega^{\star}}\right)^{2} \int_{0}^{1} \frac{d t}{1+\left(\omega / \omega^{\star}\right)^{6} t^{2}\left(3-2 t^{2}\right)}
$$

with

$$
\omega^{\star}=\sqrt{3} A^{1 / 6}(\delta f)^{1 / 3} / \sqrt{M}
$$

and after integration finally Eq. (1)is obtained.

For small frequencies, below the Boson peak, $\omega \ll \omega_{b}$ only small random forces $f$ contribute to the second integral in Eq. (20). In this case the distribution function $P(f)$ can be approximated by a constant value $P(0)$ and we get from Eq. (20)

$$
g(\omega) \propto \omega^{3} \int_{0}^{\omega} d \omega_{1} \frac{\omega_{1}}{\sqrt{\omega^{2}-\omega_{1}^{2}}} \propto \omega^{4} .
$$

As a result at low frequencies the renormalized DOS is $g(\omega) \propto \omega^{4} .{ }^{47}$ For sufficiently large frequencies $\omega \gg \omega_{b}$ the action of random static forces on the $\mathrm{HO}$ spectrum can be discarded. In this case we recover the linear DOS $g(\omega)$ $\propto \omega$. 
The BP frequency $\omega_{b} \approx 1.1 \omega^{\star}$ is determined by the characteristic value of the random static force $\delta f$, Eq. (16), acting on an $\mathrm{HO}$ with the characteristic frequency $\omega_{c}$. According to Eq. (16), it is due to the interaction between HO's with frequencies of order of $\omega_{c}$, i.e.,

$$
I_{i j}^{(c)} \approx J n_{c}, \quad J \approx I / n_{0} \approx M \omega_{c} / n_{0} g_{0}\left(\omega_{0}\right),
$$

where $n_{c} \approx n_{0} g_{0}\left(\omega_{c}\right) \omega_{c}$ is the concentration of these HO's. The characteristic displacement of a low-frequency HO's (13) from the equilibrium position is $x_{j 0} \approx \omega_{c} \sqrt{M / A}$. As a result, we get the estimate

$$
\delta f \approx M \sqrt{\frac{M}{A}} \omega_{c}^{3} \frac{g_{0}\left(\omega_{c}\right)}{g_{0}\left(\omega_{0}\right)}
$$

and according to Eq. (23)

$$
\omega_{b} \approx \omega_{c}\left[g_{0}\left(\omega_{c}\right) / g_{0}\left(\omega_{0}\right)\right]^{1 / 3}, \quad \omega_{b} \ll \omega_{c} .
$$

Again, in lowest order the anharmonicity $A$ does not enter this formula.

As a result we get a following estimate for the reconstructed DOS:

$$
g(\omega) \simeq \begin{cases}g_{0}(\omega), & \omega>\omega_{c}, \\ \omega g_{0}\left(\omega_{c}\right) / \omega_{c}, & \omega_{b}<\omega<\omega_{c}, \\ \omega^{4} g_{0}\left(\omega_{0}\right) / \omega_{c}^{4}, & \omega<\omega_{b} .\end{cases}
$$

If the DOS of the noninteracting oscillators is given by a power law $g_{0}(\omega) \propto \omega^{n}$, the BP frequency $\omega_{b}$ scales with the interaction strength $I$ as

$$
\omega_{b} \propto|I|^{1+n / 3} .
$$

Since in accordance with Eq. (8) $\omega_{c} \propto|I|$, we have always $\omega_{b} \ll \omega_{c}$ for $n>0$.

\section{NUMERICAL SIMULATION}

To test our ideas by numerical simulations, we placed $N$ oscillators with frequencies $0<\omega_{i}<1$ on a simple cubic lattice with lattice constant $a=1$ and periodic boundary conditions. To simulate random orientations of the oscillators we took for $g_{i j}$, Eq. (4), random numbers in the interval [-0.5,0.5]. The masses $M_{i}$ and anharmonicity parameters $A_{i}$ were put to 1 . The DOS for the noninteracting oscillators was taken as $g_{0}(\omega) \propto \omega^{n}$, with $n=1,2,3$.

Using the potential energy given by the generalization of Eq. (3) plus the anharmonicity [Eq. (7)] we then minimized the potential energy, and in the usual harmonic expansion around this minimum calculated the DOS for different interaction strengths $J$. This was repeated for up to 10000 representations. To check for size dependence we did the calculations for different $N$. Apart from the case $J=0.07$ the results did not change between $N=2097$ and $N=4096$.

The predicted change over in the $\omega$ dependence of the DOS at two characteristic frequencies $\omega_{c}$ and $\omega_{b}$ and the linear part in between can be clearly observed in a log-log representation, Fig. 2, for $g_{0}(\omega) \propto \omega^{2}$ and $J=0.1$. From the calculated eigenvectors we find that, as expected, at the low-

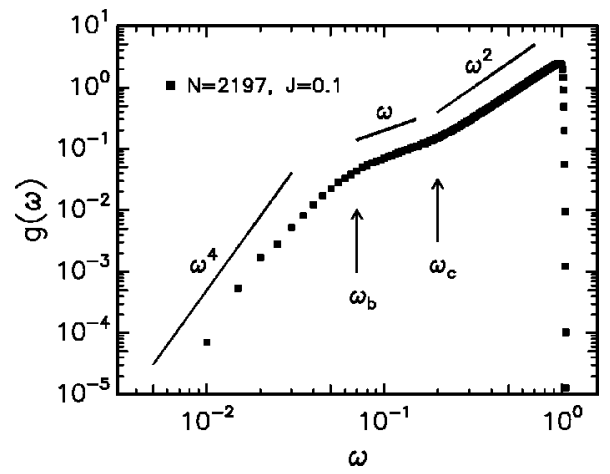

FIG. 2. Simulated density of states $\left[g_{0}(\omega) \propto \omega^{2}, N=2097\right]$ in a $\log -\log$ representation. The arrows indicate the two characteristic frequencies $\omega_{b}$ and $\omega_{c}$.

est frequencies the $\mathrm{HO}$ are weakly coupled whereas near and above $\omega_{b}$ the eigenmodes are complicated superpositions of many HO's.

Figure 3 shows the dependence of the simulated $g(\omega) / \omega^{2}$ on the interaction strength $J$. We can see the general increase of $\omega_{b}$ and related decrease of the BP intensity with increasing $J$. Our simulations cover one decade in BP frequencies. The insert shows that, in full agreement with our predictions [see Eqs. (8) and (28)], the crossover frequencies change with interaction $J$ as $\omega_{c} \propto J$ and $\omega_{b} \propto J^{1+n / 3}$.

\section{DISCUSSION AND COMPARISON WITH EXPERIMENT}

In Fig. 4 we compare our theoretical curve, Eq. (1), with Raman scattering data of lithium borate glasses ${ }^{55}$ with different compositions. The agreement is remarkably good over the whole composition range. This supports the idea of a universal shape of the Boson peak. ${ }^{56}$ The shift of the BP to higher frequencies with increasing concentration of $\mathrm{Li}_{2} \mathrm{O}$ can be explained by an increase of the total concentration of QLV's and consequently of their interaction.

The agreement between theory and experiment is not confined to this class of material. This is exemplified by Fig. 5 which shows a comparison of the theoretical curve with numerical simulation results and neutron and Raman scattering

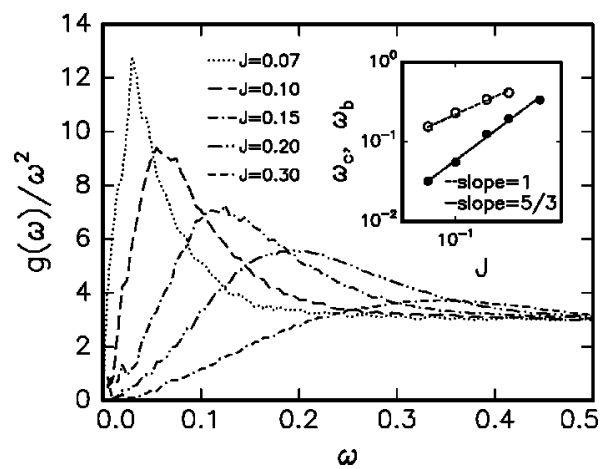

FIG. 3. Simulated $g(\omega) / \omega^{2}$ for different interaction strengths $\left[g_{0}(\omega) \propto \omega^{2}, N=2097\right.$, and $\left.N=4096(J=0.07)\right]$. The inset shows the scaling of the crossover frequencies $\omega_{c}(\bigcirc)$ and $\omega_{b}(\bigcirc)$ with interaction strength $J$. 


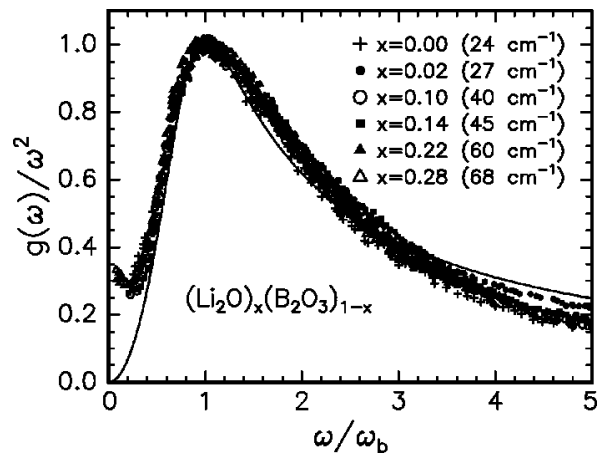

FIG. 4. Boson peak in reduced units: Eq. (1) (solid line) and Raman data for lithium borate glasses (Ref. 55). The positions of the Boson peak (for different compositions $x$ ) are given in brackets.

data for three different glasses.

One of the most important results of our theory is the predicted linear frequency dependence of the density of vibrational states above the Boson peak. It stems from the vibrational instability of interacting harmonic modes. Such linear behavior has been observed in many numerical simulations on different glasses and model disordered systems. ${ }^{32,35,36,39,58,59}$ It is also in a good agreement with many experimental results ${ }^{60,57,61-66}$ where the vibrational DOS has a section with near linear frequency dependence. Figure 6 shows this for vitreous silica at different temperatures. Above the Boson peak the DOS increases approximately linearly with frequency.

In this paper we dealt with the case of weak interaction between HO's. If the interaction is increased the characteristic frequencies $\omega_{b}$ and $\omega_{c}$ grow and the gap between them narrows and finally disappears. Then our "BP" in $g(\omega) / \omega^{2}$ superimposes the "boundary peak" in $g_{0}(\omega)$ at the edge of the assumed spectrum of naked (noninteracting) QLV's. The BP can no longer be distinguished from the boundary peak or from a possible equivalent maximum in $g_{0}(\omega)$. It is possible that in some cases, e.g., orientational glasses, $g_{0}(\omega)$ has a pronounced peak which is still visible after the reshaping of the DOS by interaction.

Similar models with strong coupling between oscillators

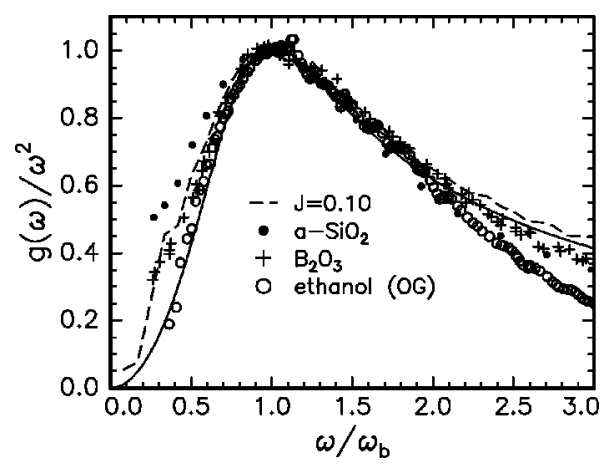

FIG. 5. Boson peak in reduced units: Eq. (1) (solid line), numerical simulation, Fig. 3 (dashed line), neutron scattering data for $a-\mathrm{SiO}_{2}$ at $T=51 \mathrm{~K}$ (Ref. 57) (O) and for the orientational glass phase of ethanol (Ref. 23) (O) and Raman data for $a-\mathrm{B}_{2} \mathrm{O}_{3}$ (Ref. 55) $(+)$.

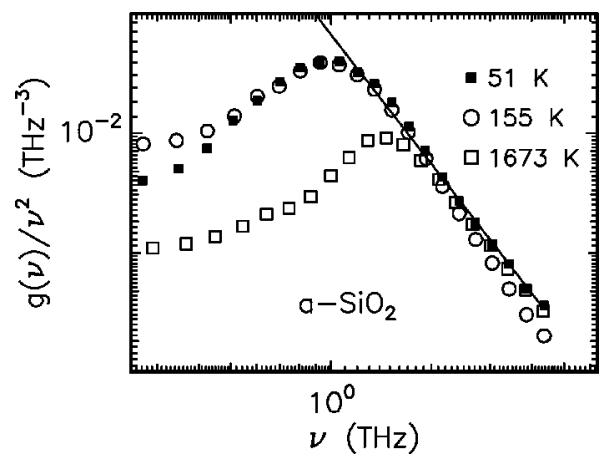

FIG. 6. Density of states of vitreous silica for three temperatures taken from inelastic neutron scattering data (Ref. 57). The slope of the straight line on the figure is equal to -1.1 .

were previously investigated by molecular dynamic simulations ${ }^{22}$ and by the replica method. ${ }^{54}$ For example, in Ref. 22 Grannan et al. assumed that the dynamics "is completely dominated by the interaction between defects mediated by the strain field." The local field (harmonic and anharmonic contribution) was neglected. We consider the opposite case where the local field (harmonic and anharmonic) dominates and interaction between dipoles is weak.

\section{CONCLUSION}

In conclusion we presented a universal picture of the BP formation in glasses. We have shown that the low frequency quasilocalized harmonic modes in glasses are destabilized by the weak bilinear interaction between them. Anharmonicity stabilizes the system in a minimum of configuration space. It completely reconstructs the low frequency part of the spectrum (at $\omega<\omega_{c}$ ) and the Boson peak feature (at $\omega_{b} \ll \omega_{c}$ ) naturally emerges. The thus created boson peak has a material independent shape. At low frequencies, below the BP, the vibrational DOS increases as $g(\omega) \propto \omega^{4}$, and above the BP, as $g(\omega) \propto \omega$.

Although the anharmonicity is responsible for this effect, the final spectrum of stable vibrations remains harmonic. A remarkable feature of the presented theory is that the strength of the anharmonicity does not enter the stable spectrum at all. It looks as if the anharmonicity does all the work, it stabilizes the system in a minimum and reconstructs the spectrum and then disappears. Therefore, the discussed phenomenon is independent of the variation of the anharmonicity between different materials. The only parameter entering the final density of states is the strength of interaction between the HO's.

Compared to previous work, the main result of our approach is the natural emergence of the $\mathrm{BP}$ on the unstructured, flat low frequency part of the initial spectrum $g_{0}(\omega) / \omega^{2}$ where the DOS previously had no peaks. For small interactions the BP frequency is much smaller than the Debye frequency value. It shifts with interaction strength $I$ which explains the large variety of BP magnitudes found in experiment. In contrast with previous models, a natural connection of the Boson peak phenomena with other universal properties of glasses is established. 


\section{ACKNOWLEDGMENTS}

The authors V.L.G. and D.A.P. gratefully acknowledge the financial support of the German Ministry of Science and Technology and the hospitality of the Forschungszentrum Jülich where part of the work was done.

\section{APPENDIX A: CLUSTER APPROACH}

Consider a cluster containing a low frequency oscillator with frequency $\omega_{1} \lesssim \omega_{c}$ surrounded by a large number $s-1$ of HO's with much higher frequencies $\omega_{j} \sim \omega_{0}$. Inclusive of their interaction, the total potential energy of the cluster is

$$
U_{\mathrm{tot}}=\sum_{i} \frac{M_{i} \omega_{i}^{2}}{2}+\frac{1}{4} \sum_{i} A_{i} x_{i}^{4}-\frac{1}{2} \sum_{i, j \neq i} I_{i j} x_{i} x_{j} .
$$

The equilibrium positions of the HO's $x_{i 0}$ are given by the system of $s$ nonlinear equations

$$
M_{i} \omega_{i}^{2} x_{i 0}+A_{i} x_{i 0}^{3}=\sum_{j \neq i} I_{i j} x_{j 0}, \quad i=1,2, \ldots, s
$$

In the case of instability $\left(x_{i 0} \neq 0\right)$, in analogy to the previously considered case of a pair of oscillators, the static displacements of the high-frequency oscillators are much smaller than the one of the low-frequency oscillator $x_{10}$. Therefore, in leading order

$$
x_{i 0}=\left(I_{1 i} / M_{i} \omega_{i}^{2}\right) x_{10}, \quad i \neq 1 .
$$

Inserting these values into Eq. (A2) for $i=1$ we get

$$
M_{1} \omega_{1}^{2} x_{10}+A_{1} x_{10}^{3}=x_{10} \sum_{i \neq 1} \frac{I_{1 i}^{2}}{M_{i} \omega_{i}^{2}}
$$

Under the condition

$$
M_{1} \omega_{1}^{2}<k \text { where } k \equiv \sum_{i \neq 1} \frac{I_{1 i}^{2}}{M_{i} \omega_{i}^{2}}
$$

the cluster becomes unstable and the low frequency oscillator is displaced to a minimum

$$
x_{10}=\sqrt{\left(k-M_{1} \omega_{1}^{2}\right) / A_{1}} .
$$

In the opposite case, $M_{1} \omega_{1}^{2}>k$, the cluster is stable and $x_{i 0}$ $=0$.

The eigenfrequencies of the interacting oscillators are the solutions of the secular equation of order of $s$

$$
\left|\begin{array}{cccc}
\alpha_{1}-M_{1} \omega^{2} & -I_{12} & \ldots & -I_{1 s} \\
-I_{21} & \alpha_{2}-M_{2} \omega^{2} & \ldots & -I_{2 s} \\
\vdots & \vdots & \ddots & \vdots \\
-I_{s 1} & -I_{s 2} & \ldots & \alpha_{s}-M_{s} \omega^{2}
\end{array}\right|=0
$$

Here the $\alpha_{i}$ are given by Eq. (11). In leading order in $I_{i j} / M \omega_{0}^{2}$ the secular equation is a linear equation for $\omega^{2}$ [compare Eq. (15)]

$$
\left(\alpha_{1}-M_{1} \omega^{2}\right) \prod_{j \neq 1} M_{j} \omega_{j}^{2}-\sum_{i \neq 1} I_{1 i}^{2} \prod_{j \neq 1, i} M_{j} \omega_{j}^{2}=0
$$

or

$$
\alpha_{1}-M_{1} \omega^{2}=\sum_{i \neq 1} \frac{I_{1 i}^{2}}{M_{i} \omega_{i}^{2}}=k
$$

and the new low frequency of the system of coupled oscillators is given by

$$
\widetilde{\omega_{1}^{2}}=\left\{\begin{array}{cc}
\frac{1}{M_{1}}\left(M_{1} \omega_{1}^{2}-k\right), & k<M_{1} \omega_{1}^{2}, \\
\frac{2}{M_{1}}\left(k-M_{1} \omega_{1}^{2}\right), & k>M_{1} \omega_{1}^{2} .
\end{array}\right.
$$

As in the case of a pair of oscillators, the anharmonicity has been used in the derivation of Eq. (A10) but does not appear in this or our final result Eq. (1).

To derive the (reconstructed) DOS the distribution of $k$, $\rho(k)$, has to be calculated. Inserting Eqs. (4) and (A5) into the definition of $\rho(k)$ gives

$$
\rho(k)=\left\langle\delta\left(k-\frac{J^{2}}{M} \sum_{j \neq 1} \frac{g_{1 j}^{2}}{r_{1 j}^{6} \omega_{j}^{2}}\right)\right\rangle .
$$

Here the angular brackets denote averaging over the positions of the $s-1$ high frequency HO's, their frequencies and orientations. For simplicity we take equal masses $M_{j}=M$ and for $g_{i j}$ a uniform distribution in the interval $[-1 / 2,1 / 2]$.

Using the Holtsmark method ${ }^{67}$ (see Appendix B) one gets

$$
\rho(k)=\frac{1}{\sqrt{2 \pi}} \frac{B}{k^{3 / 2}} \exp \left(-\frac{B^{2}}{2 k}\right)
$$

where

$$
B=\frac{\pi}{3} \sqrt{\frac{\pi}{2}} \frac{J n_{0}}{\sqrt{M}}\left\langle\frac{1}{\omega}\right\rangle_{0} \equiv \omega_{c} \sqrt{M} .
$$

Here $n_{0}$ is the total concentration of HO in the cluster and $\langle 1 / \omega\rangle_{0}$ is the $\omega^{-1}$ moment of the normalized initial DOS $g_{0}(\omega)$. This formula is a more accurate definition of the characteristic frequency $\omega_{c}$ introduced in Eq. (8). Note that the distribution $\rho(k)$ (A12) belongs to an important class of one-sided stable distributions, Ref. 68.

Due to the combined action of interaction and anharmonicity the DOS is reconstructed to $\widetilde{g}(\omega)=2 \omega \widetilde{G}\left(\omega^{2}\right)$ with

$$
\begin{aligned}
\widetilde{G}\left(\omega^{2}\right) & =\left\langle\delta\left(\omega^{2}-\widetilde{\omega}_{1}^{2}\right)\right\rangle_{k, \omega_{1}} \\
& \equiv \int_{0}^{\infty} d k \rho(k) \int_{0}^{\infty} d \omega_{1}^{2} G_{0}\left(\omega_{1}^{2}\right) \delta\left(\omega^{2}-\tilde{\omega}_{1}^{2}\right)
\end{aligned}
$$

and $G_{0}\left(\omega_{1}^{2}\right) \equiv g_{0}\left(\omega_{1}\right) / 2 \omega_{1}$. Using Eq. (A10) and integrating Eq. (A14) we obtain 


$$
\begin{aligned}
\widetilde{G}\left(\omega^{2}\right)= & \frac{1}{2} \int_{0}^{\infty} d k \rho\left(k+\frac{1}{2} M \omega^{2}\right) G_{0}\left(\frac{k}{M}\right) \\
& +\int_{0}^{\infty} d k \rho(k) G_{0}\left(\omega^{2}+\frac{k}{M}\right) .
\end{aligned}
$$

For low frequencies, $\omega \ll \omega_{c}, \widetilde{G}\left(\omega^{2}\right)=$ const and $\widetilde{g}(\omega) \propto \omega$, i.e., the reconstructed DOS is a linear function of $\omega$. For high frequencies the first term in Eq. (A15) can be discarded and the original DOS is reproduced, $\widetilde{G}\left(\omega^{2}\right)=G_{0}\left(\omega^{2}\right)$ for $\omega \gg \omega_{c}$.

\section{APPENDIX B: HOLTSMARK METHOD}

\section{Distribution of random forces}

Let $x_{i}$ be a random value with zero mean $\langle x\rangle=0$ and finite $\langle|x|\rangle$ and let $\mathbf{r}_{i}(N \rightarrow \infty)$ be Poisson-distributed random points in three-dimensional (3D) space with concentration $n_{c}$. The distribution function $P(f)$ of the random values

$$
f=\sum_{i} \frac{x_{i}}{r_{i}^{3}}
$$

can then be calculated by the Holtsmark method. ${ }^{67}$

$$
P(f)=\left\langle\delta\left(f-\sum_{i} \frac{x_{i}}{r_{i}^{3}}\right)\right\rangle \equiv \frac{1}{2 \pi} \int_{-\infty}^{\infty} d \tau e^{i f \tau} F(\tau)
$$

with

$$
F(\tau)=\left\langle\exp \left[-i \tau \sum_{i} \frac{x_{i}}{r_{i}^{3}}\right]\right\rangle
$$

where angular brackets denote averaging over $x_{i}$ and $\mathbf{r}_{i}$. Since the values $x_{i} / r_{i}^{3}$ are independent of each other

$$
F(\tau)=\left\langle e^{-i \tau x / r^{3}}\right\rangle^{N}=\left(1-\frac{1}{V} \int d^{3} \mathbf{r}\left\langle 1-e^{-i \tau x / r^{3}}\right\rangle_{x}\right)^{N}
$$

which in the limit $N \rightarrow \infty$ becomes

$$
F(\tau)=\exp \left[-n_{c} \int d^{3} \mathbf{r}\left\langle 1-e^{-i \tau x / r^{3}}\right\rangle_{x}\right]
$$

Using $\langle x\rangle>=0$ and changing the integration variable to $y$ $=|\tau||x| / r^{3}$ we get

$$
F(\tau)=\exp \left[-\frac{4 \pi n_{c}}{3}|\tau|\langle|x|\rangle \int_{0}^{\infty} \frac{d y}{y^{2}}(1-\cos y)\right] .
$$

The integral equals $\pi / 2$ and Eq. (B2) is the Fourier transform of a Lorentzian distribution of random forces

$$
P(f)=\frac{1}{\pi} \frac{\delta f}{f^{2}+(\delta f)^{2}}
$$

where the width of the distribution is given by

$$
\delta f=\frac{2 \pi^{2} n_{c}}{3}\langle|x|\rangle
$$

\section{2. $\rho(k)$ distribution}

The same method can be applied to calculate the distribution of the random quantity $k$

$$
k=\sum_{i} \frac{x_{i}^{2}}{r_{i}^{6} \omega_{i}^{2}}
$$

where the $x_{i}$ 's are random and uniformly distributed, $-x_{0} / 2<x_{i}<x_{0} / 2, \mathbf{r}_{i}$ are $(N \rightarrow \infty)$ Poisson-distributed random points in $3 \mathrm{D}$ space (concentration $n_{0}$ ) and $\omega_{i}$ are random frequencies of HO's distributed in the interval

$$
0<\omega_{i}<\omega_{0}
$$

with a DOS $g_{0}(\omega)$ normalized to unity.

Analogously to Eq. (B2) the distribution $\rho(k)$ can be written as

$$
\rho(k)=\frac{1}{2 \pi} \int_{-\infty}^{\infty} d \tau e^{i k \tau} K(\tau)
$$

with

$$
K(\tau)=\left\langle\exp \left[-i \tau \sum_{i} \frac{x_{i}^{2}}{r_{i}^{6} \omega_{i}^{2}}\right]\right\rangle
$$

Following the steps of the previous subsection we can write

$$
K(\tau)=\exp \left[-n_{0} \int d^{3} \mathbf{r}\left\langle 1-e^{-i \tau x^{2} / r^{6} \omega^{2}}\right\rangle_{x, \omega}\right]
$$

and, introducing the new variable $y=\left(x^{2}|\tau|\right) /\left(r^{6} \omega^{2}\right)$,

$$
K(\tau)=\exp \left[-\frac{2 \pi}{3} n_{0}\left\langle\frac{|x|}{\omega}\right\rangle_{x, \omega} \sqrt{|\tau|}(\alpha+i \beta \operatorname{sgn} \tau)\right]
$$

where

$$
\alpha=\int_{0}^{\infty} \frac{d y}{y^{3 / 2}}(1-\cos y), \quad \beta=\int_{0}^{\infty} \frac{d y}{y^{3 / 2}} \sin y .
$$

It is straightforward to show that $\alpha=\beta=\sqrt{2 \pi}$. Therefore

$$
K(\tau)=\exp [-B \sqrt{|\tau|}(1+i \operatorname{sgn} \tau)]
$$

with

$$
B=\frac{\pi}{3} \sqrt{\frac{\pi}{2}} n_{0} x_{0}\left\langle\frac{1}{\omega}\right\rangle .
$$

After integration in Eq. (B11) with $K(\tau)$ from Eq. (B16) we finally get

$$
\rho(k)=\frac{1}{\sqrt{2 \pi}} \frac{B}{k^{3 / 2}} \exp \left(-\frac{B^{2}}{2 k}\right)
$$


${ }^{1}$ Amorphous Solids. Low Temperature Properties, edited by W. A. Phillips (Springer-Verlag, Berlin, 1981).

${ }^{2}$ N. Ahmad, K. W. Hutt, and W. A. Phillips, J. Phys. C 19, 3765 (1986)

${ }^{3}$ V. K. Malinovsky, V. N. Novikov, and A. P. Sokolov, Phys. Lett. A 153, 63 (1991).

${ }^{4}$ T. Pang, Phys. Rev. B 45, 2490 (1992).

${ }^{5}$ S. R. Elliott, Europhys. Lett. 19, 201 (1992).

${ }^{6}$ C. Masciovecchio, V. Mazzacurati, G. Monaco, G. Ruocco, T. Scopigno, F. Sette, P. Benassi, A. Consulo, A. Fontana, M. Kirsch, A. Mermet, M. Montagna, F. Rossi, M. Sampoli, G. Signorelli, and R. Verbeni, Philos. Mag. B 79, 2013 (1999).

${ }^{7}$ S. N. Taraskin and S. R. Elliott, Phys. Rev. B 61, 12017 (2000).

${ }^{8}$ J. A. Freitas, Jr., B. V. Shanabrook, and U. Strom, J. Non-Cryst. Solids 77/78, 1125 (1985).

${ }^{9}$ M. T. Dove, M. J. Harris, A. C. Hannon, J. M. Parker, I. P. Swainson, and M. Gambhir, Phys. Rev. Lett. 78, 1070 (1997).

${ }^{10}$ V. N. Sagaev, E. N. Smelyanskaya, V. G. Plotnichenko, V. V. Koltashev, A. A. Volkov, and P. Pernice, J. Non-Cryst. Solids 248, 141 (1999).

${ }^{11}$ W. Schirmacher, G. Diezemann, and C. Ganter, Phys. Rev. Lett. 81, 136 (1998).

${ }^{12}$ S. N. Taraskin, Y. L. Loh, G. Natarajan, and S. R. Elliott, Phys. Rev. Lett. 86, 1255 (2001).

${ }^{13}$ J. W. Kantelhardt, S. Russ, and A. Bunde, Phys. Rev. B 63, 064302 (2001).

${ }^{14}$ T. S. Grigera, V. Martin-Mayor, G. Parisi, and P. Verrocchio, J. Phys.: Condens. Matter 14, 2167 (2002).

${ }^{15}$ V. G. Karpov and D. A. Parshin, JETP Lett. 38, 648 (1983); Sov. Phys. JETP 61, 1308 (1985).

${ }^{16}$ M. A. Krivoglaz, Sov. Phys. JETP 61, 1284 (1985).

${ }^{17}$ U. Buchenau, N. Nücker, and A. J. Dianoux, Phys. Rev. Lett. 53, 2316 (1984).

${ }^{18}$ A. A. Maradudin, E. W. Montroll, G. H. Weiss, and I. P. Ipatova, Solid State Physics, 2nd ed. (Academic Press, New York, 1971), Suppl. 3.

${ }^{19}$ P. H. Dederichs and R. Zeller, in Point Defects in Metals II, Vol. 87 of Springer Tracts in Modern Physics (Springer-Verlag, Berlin, 1980)

${ }^{20}$ U. Buchenau, Yu. M. Galperin, V. L. Gurevich, D. A. Parshin, M. A. Ramos, and H. R. Schober, Phys. Rev. B 46, 2798 (1992).

${ }^{21}$ C. C. Yu and A. J. Leggett, Comments Condens. Matter Phys. 14, 231 (1988); A. J. Leggett, Physica B 169, 322 (1991).

${ }^{22}$ E. R. Grannan, M. Randeria, and J. P. Sethna, Phys. Rev. B 41, 7799 (1990).

${ }^{23}$ M. A. Ramos, S. Vieira, F. J. Bermejo, J. Dawidowski, H. E. Fischer, H. Schober, M. A. González, C. K. Loong, and D. L. Price, Phys. Rev. Lett. 78, 82 (1997).

${ }^{24}$ R. M. Lynden-Bell and K. H. Michel, Rev. Mod. Phys. 66, 721 (1994).

${ }^{25}$ The Plastically Crystalline State (Orientationally-Disordered Crystals), edited by J. N. Sherwood (Wiley, New York, 1978).

${ }^{26}$ P. Lunkenheimer, R. Brand, R. Wehn, and A. Loidl, cond-mat/0210067 (unpublished).

${ }^{27}$ R. Biswas, A. M. Bouchard, W. A. Kamitakahara, G. S. Grest, and C. M. Soukoulis, Phys. Rev. Lett. 60, 2280 (1988).

${ }^{28}$ J. Fabian and P. B. Allen, Phys. Rev. Lett. 79, 1885 (1997); J. Fabian and P. B. Allen, ibid. 82, 1478 (1999).

${ }^{29}$ J. L. Feldman, C. S. Hellberg, G. Viliani, W. Garber, and F. M.
Tangerman, Philos. Mag. 82, 133 (2002).

${ }^{30}$ P. H. Dederichs, C. Lehmann, H. R. Schober, A. Scholz, and R. Zeller, J. Nucl. Mater. 69/70, 176 (1978).

${ }^{31}$ A. V. Granato, Phys. Rev. Lett. 68, 974 (1992).

${ }^{32}$ B. B. Laird and H. R. Schober, Phys. Rev. Lett. 66, 636 (1991); H. R. Schober and B. B. Laird, Phys. Rev. B 44, 6746 (1991).

${ }^{33}$ W. Jin, P. Vashishta, R. K. Kalia, and J. P. Rino, Phys. Rev. B 48, 9359 (1993).

${ }^{34}$ C. Oligschleger and H. R. Schober, Physica A 201, 391 (1993).

${ }^{35}$ J. Hafner and M. Krajčí, J. Phys.: Condens. Matter 6, 4631 (1994).

${ }^{36}$ P. Ballone and S. Rubini, Phys. Rev. B 51, 14962 (1995).

${ }^{37}$ L. D. V. Ee, B. J. Thijsse, and J. Sietsma, J. Non-Cryst. Solids 205-207, 641 (1996).

${ }^{38}$ M. Cho, G. R. Fleming, S. Saito, I. Ohmine, and R. M. Stratt, J. Chem. Phys. 100, 6672 (1994).

${ }^{39}$ J. Hafner and M. Krajčí, J. Phys.: Condens. Matter 5, 2489 (1993).

${ }^{40}$ Ten-Ming Wu, Wen-Jong Ma, and S. L. Chang, J. Chem. Phys. 113, 274 (2000).

${ }^{41}$ H. R. Schober and C. Oligschleger, Phys. Rev. B 53, 11469 (1996).

${ }^{42}$ V. A. Luchnikov, N. N. Medvedev, Yu. I. Naberukhin, and H. R. Schober, Phys. Rev. B 62, 3181 (2000).

${ }^{43}$ S.-P. Chen, T. Egami, and V. Vitek, Phys. Rev. B 37, 2440 (1988).

${ }^{44}$ H. R. Schober, C. Oligschleger, and B. B. Laird, J. Non-Cryst. Solids 156-158, 965 (1993).

${ }^{45}$ C. Oligschleger, Phys. Rev. B 60, 3182 (1999).

${ }^{46}$ V. G. Karpov, M. I. Klinger, and F. N. Ignatiev, Sov. Phys. JETP 57, 439 (1983).

${ }^{47}$ M. A. Il'in, V. G. Karpov, and D. A. Parshin, Sov. Phys. JETP 65, 165 (1987).

${ }^{48}$ D. A. Parshin, Sov. Phys. Solid State 36, 991 (1994).

${ }^{49}$ U. Buchenau, Y. M. Galperin, V. L. Gurevich, and H. R. Schober, Phys. Rev. B 43, 5039 (1991).

${ }^{50}$ D. A. Parshin, X. Liu, O. Brand, and H. v. Löhneysen, Z. Phys. B: Condens. Matter 93, 57 (1993).

${ }^{51}$ G. D'Angelo, G. Carini, G. Tripodo, A. Bartolotta, G. Di Marco, and G. Salvato, Philos. Mag. B 82, 331 (2002).

${ }^{52}$ M. A. Ramos and U. Buchenau, Phys. Rev. B 55, 5749 (1997).

${ }^{53}$ V. L. Gurevich, D. A. Parshin, J. Pelous, and H. R. Schober, Phys. Rev. B 48, 16318 (1993).

${ }^{54}$ R. Kühn and U. Horstmann, Phys. Rev. Lett. 78, 4067 (1997).

${ }^{55}$ S. Kojima and M. Kodama, Physica B 263, 336 (1999).

${ }^{56}$ V. K. Malinovsky and A. P. Sokolov, Solid State Commun. 57, 757 (1986); V. K. Malinovsky, V. N. Novikov, P. P. Parshin, A. P. Sokolov, and M. G. Zemlyanov, Europhys. Lett. 11, 43 (1990).

${ }^{57}$ A. Wischnewski, U. Buchenau, A. J. Dianoux, W. A. Kamitakahara, and J. L. Zarestky, Philos. Mag. B 77, 579 (1998).

${ }^{58}$ C. Oligschleger and J. C. Schön, J. Phys.: Condens. Matter 9, 1049 (1997).

${ }^{59}$ S. V. Meshkov, Phys. Rev. B 55, 12113 (1997).

${ }^{60}$ Y. Inamura, M. Arai, O. Yamamuro, A. Inaba, N. Kitamura, T. Otomo, T. Matsuo, S. M. Bennington, and A. C. Hannon, Physica B 263-264, 299 (1999).

${ }^{61}$ R. L. Cappelletti, M. Cobb, D. A. Drabold, and W. A. Kamitakahara, Phys. Rev. B 52, 9133 (1995).

${ }^{62}$ J. M. Carpenter and D. L. Price, Phys. Rev. Lett. 54, 441 (1985); 
D. L. Price and J. M. Carpenter, J. Non-Cryst. Solids 92, 153 (1987).

${ }^{63}$ W. A. Phillips, U. Buchenau, N. Nücker, A.-J. Dianoux, and W. Petry, Phys. Rev. Lett. 63, 2381 (1989).

${ }^{64}$ F. J. Bermejo, A. Criado, J. L. Martínez, E. Enciso, and B. Fåk, Physica A 201, 286 (1993).
${ }^{65}$ N. Ahmad, Phys. Rev. B 48, 13512 (1993).

${ }^{66}$ J.-B. Suck, J. Non-Cryst. Solids 293-295, 370 (2001).

${ }^{67}$ S. Chandrasekhar, Rev. Mod. Phys. 15, 1 (1943).

${ }^{68}$ W. Feller, An Introduction to Probability Theory and its Applications (Wiley, New York, 1966), Vol. 2. 